\title{
Predictive Factors for In-Hospital Preoperative Rupture in Hyperacute Type A Aortic Dissection
}

\author{
Xinlong Tang ${ }^{1,2,3}$, Yi Jiang ${ }^{1,3}$, Yunxing Xue ${ }^{1,2,3}$, Wei Chen ${ }^{1,3}$, Haitao Zhang ${ }^{1,3}$, Yuzhou Lu ${ }^{1,2,3}$, \\ He Zhang ${ }^{1,3}$, Wei Xie ${ }^{1,3}$, Bo Dai ${ }^{3}$, Qing Zhou ${ }^{1,2,3}$, Jun Pan ${ }^{1,2,3}$, Dongjin Wang, \\ ${ }^{1}$ Nanjing Drum Tower Hospital Clinical College of Nanjing Medical University, Nanjing China; ${ }^{2}$ Department of Thoracic and \\ Cardiovascular Surgery, the Affiliated Drum Tower Hospital of Nanjing University Medical School, Nanjing, China; ${ }^{3}$ Institute of \\ Cardiothoracic Vascular Disease, Nanjing University, Nanjing China
}

\section{ABSTRACT}

Objective: This study aims to figure out risk factors of in-hospital preoperative rupture of hyperacute type A aortic dissection (haTAAD) patients and build a prediction and risk stratification model.

Methods: From January 2011 to December 2019, 830 patients diagnosed as haTAAD from Nanjing Drum Tower Hospital were enrolled. Among them, 799 patients received prompt surgery and 31 suffered aortic rupture before operation. The association between in-hospital preoperative rupture and perioperative parameters were examined. Best subset selection was used for feature selection and ROC curve was used to identify the model.

Results: Age, winter season, back pain, preoperative hypotension, albumin and globulin ratio, high serum phosphorus level are risk factors for in-hospital preoperative rupture of haTAAD. On the basis of six variables with AUC 0.828, a nomogram was established. According to the robustness test, actual in-hospital preoperative ruptures were fitted well.

Conclusions: The in-hospital rupture prediction model was developed using logistic regression analysis. High serum phosphorus level is one of the strongest predictors. This nomogram may be useful when evaluating the risk of aortic dissection in-hospital rupture in future trials.

\section{INTRODUCTION}

Hyperacute type A aortic dissection (haTAAD), which refers to the onset within 48 hours, is a lethal and emergency state [Mussa 2016; Coady 1999]. Timely operation is effective and safe for most patients. However, some patients do not receive timely surgical treatment when they have been admitted in hospital. Preoperative aortic rupture is one of the main reasons. In China, most prefecture-level cities do not have a hospital capable of performing effective operation for haTAAD. Therefore, it is necessary to transfer haTAAD patients to a centralized hospital with surgical capabilities. Although there is a risk

Received March 4, 2021; accepted March 15, 2021.

Correspondence: Dongjin Wang, MD, 321 Zhongshan Road, Nanjing 210000, Fiangsu, China (e-mail: dongjinwang_gl@163.com). of transport, it also has been shown to be effective in improving the success rate of haTAAD treatment [A 2018; Goldstone 2019]. Therefore, for centralized hospitals, they often are faced with the situation that they need to receive a large number of haTAAD patients at the same time [Vincent].

To treat the aforementioned situation, we aim to make a risk stratification for haTAAD patients. We hope to identify the patients, who are more dangerous than others and give them treatment priority. There already are some studies in postoperative mortality prediction of TAAD patients, but few in the risk factors of in-hospital preoperative rupture in TAAD patients, especially in haTAAD patients. Thus, this study aims to identify predictors for in-hospital preoperative rupture in haTAAD patients, to help physicians for optimal arrangement and management.

\section{METHODS}

\section{Patients}

A retrospective cohort analysis was performed on patients presenting with haTAAD at Nanjing Drum Tower Hospital in China between January 2011 and December 2019. The institutional review board and ethics committees of both hospitals reviewed and approved the study. A total of 830 patients were divided into the rupture group $(\mathrm{N}=31)$ and non-rupture group $(\mathrm{N}=799)$.

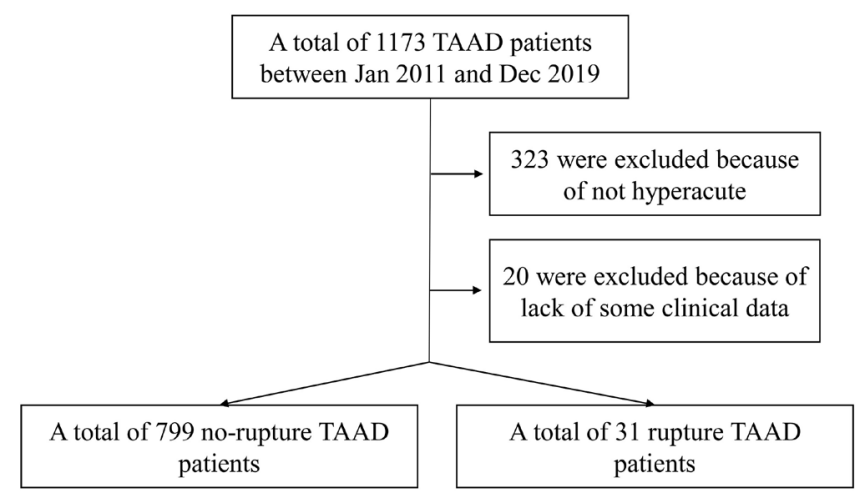

Figure 1. Flow chart of patient enrollment and exclusion. 
Table 1. Baseline characteristics of patients in the rupture group and non-rupture group

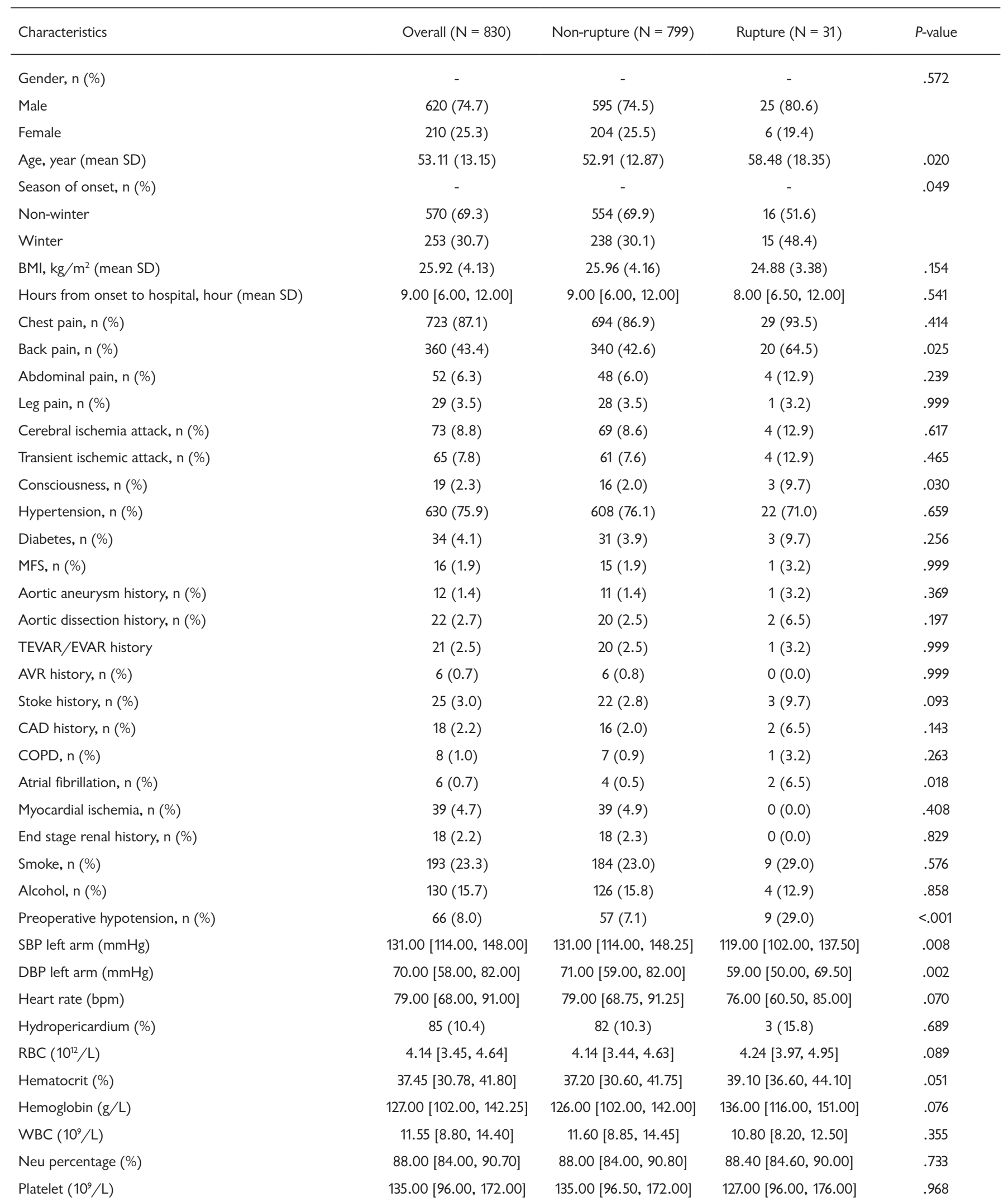


Table 1. [CONT.]

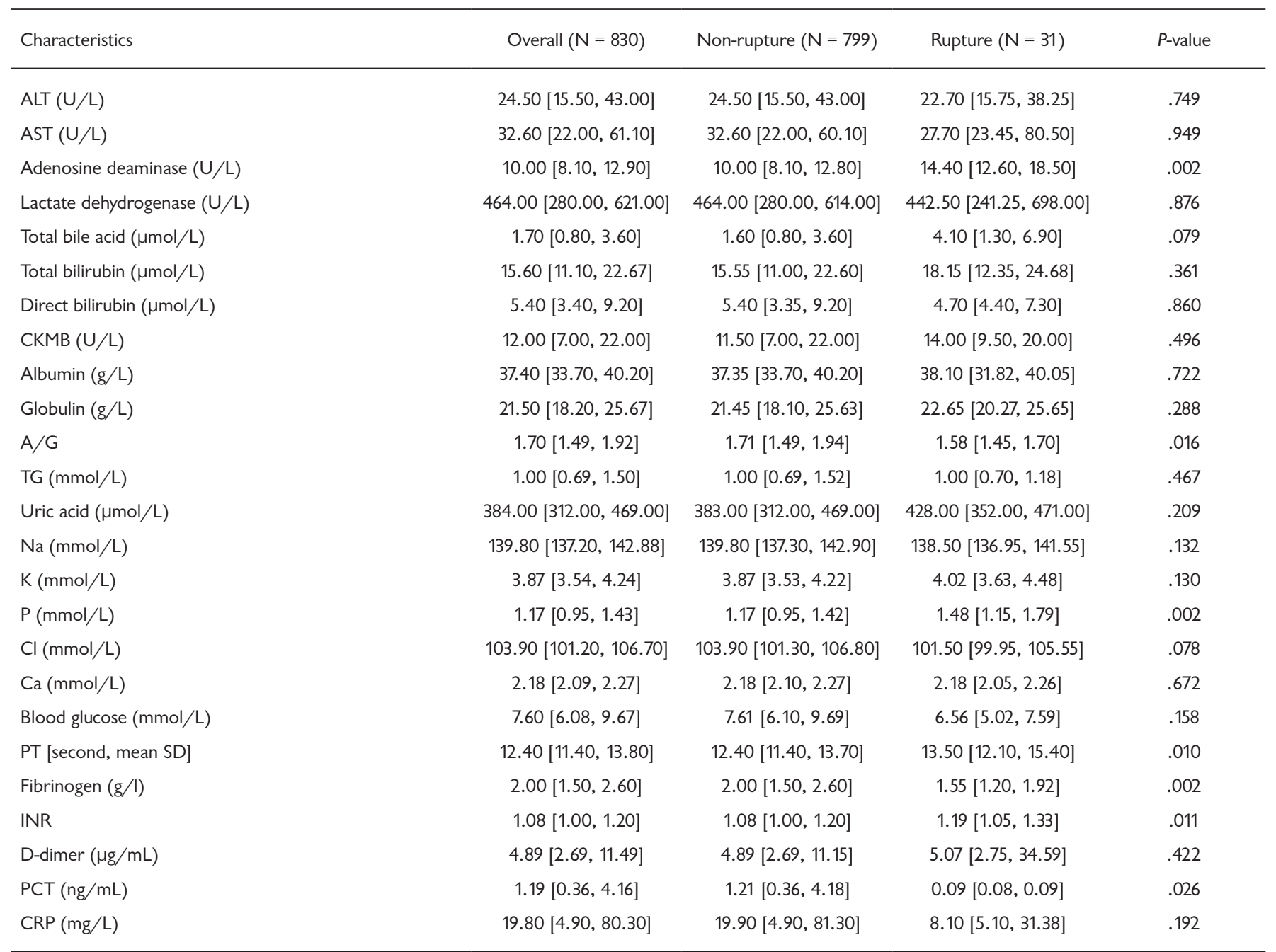

SD, standard deviation; BMI, body mass index; BP, blood pressure; CAD, coronary artery disease; EVAR, endovascular repair; AVR, aortic valve replacement; MFS, Marfan syndrome; COPD, chronic obstructive pulmonary disease; WBC, white blood cell count; RBC, red blood cell count; Neu, neutrophile granulocyte; ALT, alanine transaminase; AST, aspartate aminotransferase; A/G, albumin and globulin ratio; TG, triglycerides; Na, serum sodium; $\mathrm{K}$, serum kalium; Cl, serum chlorine; P, serum phosphorus; Ca, serum calcium; PT, prothrombin time; INR, international normalized ratio; CRP, C-reactive protein

Diagnosis of TAAD was confirmed by the presence of an intimal flap on enhanced CT scan, haTAAD was referred to the interval between the onset and admission within 48 hours and rupture was confirmed based on bedside transthoracic ultrasound. The data collected included clinical information, laboratory tests, imaging findings, and patient outcomes.

The study was approved by the institutional review board of Nanjing Drum Tower Hospital (2020-185-01).

Statistical analysis: All data are presented as N (\%) for categorical variables and mean \pm standard deviation for normally distributed continuous variables. Normality distribution were tested with the Kolmogorov-Smirnov test. Independent $\mathrm{t}$-tests were performed for normally distributed
variables,orMann-WhitneyUtestsfornon-normaldistribution. Categorical variables were analyzed by Chi-square test or Fisher's exact test, as appropriate. Multivariate analysis was performed using a binary logistic regression model after best subset feature selection to discriminate independent risk factors for in-hospital rupture.

$\mathrm{R}$ software (version 4.0.3) was used for data analysis. $\mathrm{R}$ packages "tableone" were used for basic statistics and to make table one. The package "glmnet" was used for logistic regression. The package "leaps" was used for best subset selection. Bootstrap was used to identify the robustness of the final model. A $P$-value of less than .05 was considered statistically significant. 
Table 2. Univariate analyses of factors associated with rupture

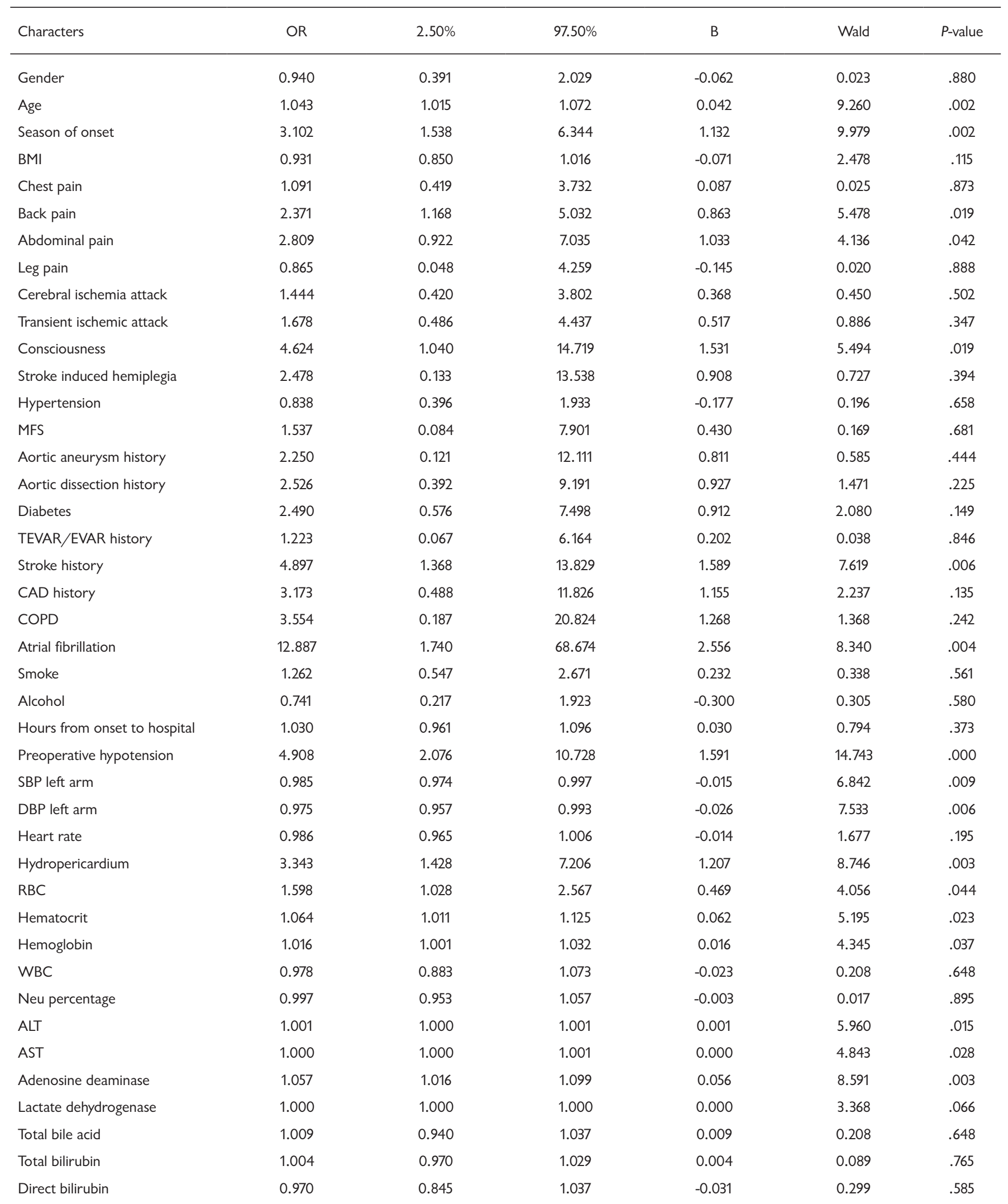


Table 2. [CONT.]

\begin{tabular}{|c|c|c|c|c|c|c|}
\hline Albumin & 0.970 & 0.906 & 1.048 & -0.030 & 0.672 & .412 \\
\hline $\mathrm{A} / \mathrm{G}$ & 0.290 & 0.094 & 0.815 & -1.238 & 5.058 & .025 \\
\hline TG & 1.032 & 0.721 & 1.308 & 0.031 & 0.045 & .833 \\
\hline $\mathrm{Na}$ & 0.924 & 0.856 & 0.990 & -0.079 & 4.461 & .035 \\
\hline K & 1.570 & 0.934 & 2.525 & 0.451 & 3.187 & .074 \\
\hline $\mathrm{Cl}$ & 0.932 & 0.857 & 1.012 & -0.070 & 2.679 & .102 \\
\hline $\mathrm{Ca}$ & 0.775 & 0.133 & 5.325 & -0.255 & 0.070 & .791 \\
\hline $\mathrm{P}$ & 3.238 & 1.736 & 5.962 & 1.175 & 14.289 & .000 \\
\hline INR & 1.167 & 0.681 & 1.559 & 0.155 & 0.759 & .384 \\
\hline D-dimer & 1.013 & 0.998 & 1.024 & 0.013 & 4.004 & .045 \\
\hline PCT & 0.000 & 0.000 & 0.015 & -16.013 & 2.084 & .149 \\
\hline CRP & 0.984 & 0.959 & 0.999 & -0.016 & 2.578 & .108 \\
\hline
\end{tabular}

BMI, body mass index; BP, blood pressure; CAD, coronary artery disease; EVAR, endovascular repair; AVR, aortic valve replacement; MFS, Marfan syndrome; COPD, chronic obstructive pulmonary disease; WBC, white blood cell count; RBC, red blood cell count; Neu, neutrophile granulocyte; ALT, alanine transaminase; AST, aspartate aminotransferase; $\mathrm{A} / \mathrm{G}$, albumin and globulin ratio; TG, triglycerides; $\mathrm{Na}$, serum sodium; $\mathrm{K}$, serum kalium; $\mathrm{Cl}$, serum chlorine; $\mathrm{P}$, serum phosphorus; Ca, serum calcium; PT, prothrombin time; INR, international normalized ratio; CRP, C-reactive protein

\section{RESULTS}

Characteristics of patients in rupture group: compared with non-rupture group

Table 1 summarizes haTAAD patients' characteristics in the total population $(\mathrm{N}=830)$. Approximately 6 percent of haTAAD patients were ruptured before or during the surgery. Compared with the non-rupture group, rupture group patients showed advanced age (60.1 versus 52.9 years, $P=$ $.001)$ and a higher rate of back pain $(63.6 \%$ versus $42.5 \%$, $P=.026)$. Patients also show a higher rate of rupture in the winter $(54.5 \%$ versus $27.9 \%, P=.002)$. However, there were no significant differences in gender and BMI between the two groups. Patients with altered consciousness and history of stroke are more prone to rupture $(9.1 \%$ versus $2.1 \%, P=.04$ and $12.1 \%$ versus $2.7 \%, P=.011$, respectively). Patients in the rupture group had lower blood pressure at admission $(27.3 \%$ versus $7.1 \%, P<.001)$, which were divided as preoperative hypotension (systolic blood pressure less than $90 \mathrm{mmHg}$ ). Hydropericardium was more common in the rupture group (27.3\% versus $10.1 \%, P=.005)$.

\section{Univariable analysis of in-bospital rupture}

Univariate analysis showed clinical features that were significantly associated with in-hospital rupture as shown in
Table 2. These included advanced age, winter season, back pain, abdominal pain, consciousness change, stroke history, atrial fibrillation history, preoperative hypotension, left arm blood pressure, hydropericardium, red blood cell, hematocrit, Hemoglobin, Aspartate Aminotransferase, adenosine deaminase, albumin and globulin ratio, uric acid, serum sodium, serum phosphorus, fibrinogen, and D-Dimer (Table 2). Advanced age and winter were the risk factors in the rupture group (OR 1.043, 95\%CI: 1.015-1.072, $P=.002$, OR 3.102, 95\% CI: $1.538-6.344, P=.002$, respectively). As the indicator to assess the condition, consciousness change (OR 4.624, 95\% CI: $1.040-14.719, P=.019)$ and preoperative hypotension (OR 4.908, 95\%CI: 2.076-10.728, $P=.000$ ) show the association with rupture. Among the biochemical indicators, high serum phosphorus (OR 3.238, 95CI\%:1.736-5.962, $P=.000)$ may be one of the important predictors.

\section{Variable selection and multivariable analysis}

Multivariate analysis was performed on the 22 indicators, which were significant in univariate analysis. Finally, six indicators were selected for modeling, such as age, back pain, albumin and globulin ratio, serum phosphorus and preoperative hypotension. As the rupture predictors, the six indicators were incorporated in multivariate regression analysis (Table 3). ROC curve was used to identify the results of multivariate regression 


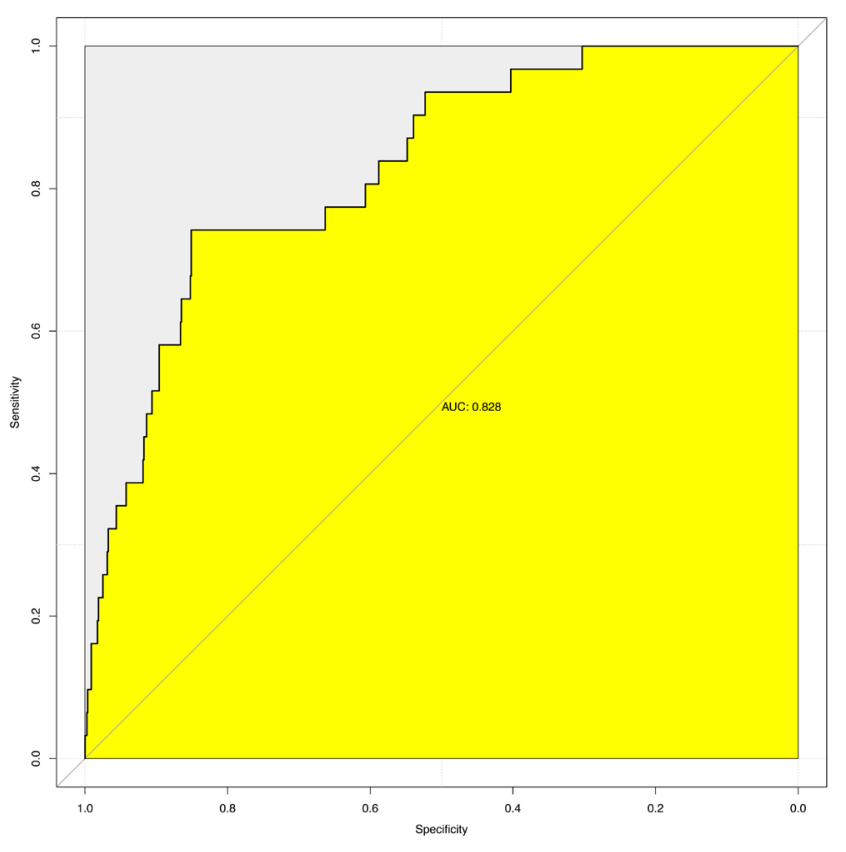

Figure 2. AUC of the model was $0.828(95 \% \mathrm{Cl}, 0.757-0.899)$. AUC, area under the curve.

and rupture (Figure 2) (Sensitivity 69.7\%, Specificity 86.2\%). The predictive nomogram also was developed from the six independent in-hospital rupture predictors (Figure 3).

\section{Robustness of the final model}

The robustness of the final model was examined by repeatedly refitting the model to 1000 differently sampled training and test sets (ratio 80:20) via the bootstrap procedure. The mean AUC is 0.791 with a $95 \%$ bootstrap CI of $0.786-0.795$.

\section{DISCUSSION}

A total of 830 patients over an eight-year period were enrolled in our study. We summarized and reported that winter season, backpain, preoperative hypotension, and high serum phosphorus level are risk factors for in-hospital rupture of haTAAD. Delaying surgery in haTAAD could never be an acceptable strategy, but those at higher risk must be considered the priority. Therefore, we should establish an objective and explicable criterion. In this retrospective analysis, we tried to find a risk stratification system based on the different factors for preoperative aortic rupture.

This study figures out several clinical variables or features that can predict in-hospital preoperative rupture in patients with haTAAD: advanced age, winter season, backpain, preoperative hypotension, low $\mathrm{A} / \mathrm{G}$ ratio, and high serum phosphorus level. A nomogram was established with a powerful discriminatory ability that easily can be used to help make a clinical decision and for patient counseling. For haTAAD patients with a high risk of in-hospital preoperative

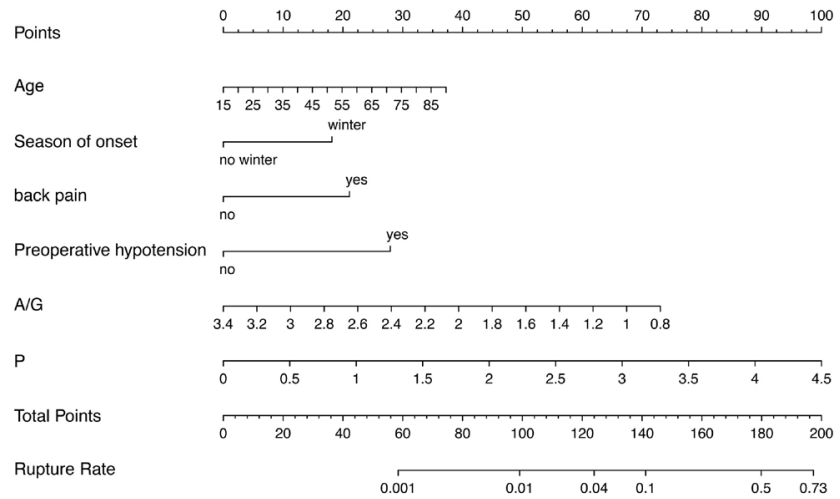

Figure 3. Nomogram for predicting the risk of preoperative rupture for in-hospital haTAAD patients.

rupture, prioritizing surgical treatment strongly should be considered.

The strongest predictor identified in our study was preoperative hypotension. We chose preoperative hypotension in the final model, and we believe pericardial effusion is highly associated with hypotension but difficult to estimate accurately in such an emergency or in some inexperienced center. The latest series report from Fuwai Hospital about inhospital rupture of TAAD introduced by $\mathrm{Wu}$ et al had revealed that hemopericardium $(P<.001)$ were associated with higher in-hospital rupture rates [Wu 2019]. Shigetaka K et al reported a novel risk score for predicting death or need for surgery in patients with acute type A intramural hematoma receiving medical therapy [Kageyama 2020]. In this risk score, SBP on arrival $<120 \mathrm{mmHg}$, pericardial effusion on admission CT, ascending aorta diameter and ulcer-like projection are risk factors involved. Cut-off threshold for the risk factors and novel risk score on admission for the prediction are SBP on arrival $<120 \mathrm{mmHg}$ with 1 point, pericardial effusion on admission CT with 1 point, ascending aorta diameter $>45 \mathrm{~mm}$ with 1 point, and ulcer-like projection with 2 point. When the threshold for the total risk score was $\geq 2$ (while $<2$ indicates that medical therapy would be acceptable), the primary endpoint could be predicted with a sensitivity of $89.7 \%$, specificity of $75 \%$ (area under the ROC of 0.823 ), and the accuracy of risk score was $80.7 \%$. According to Rampoldi et al, they described simple risk models to predict surgical mortality in acute type A aortic dissection and suggested that age $>70$, presenting hypotension/shock and preoperative cardiac tamponade are variables in their Preoperative Prediction Model [Rampoldi 2007]. What's more, age $>70$ years and presenting hypotension/shock also are involved in Prediction Model With Variables During Operation. Advanced age also is an important risk factor of rupture or in-hospital death in aortic dissection. Leontyev and colleagues demonstrated that in a risk-Adjusted Predictive Model for In-Hospital Death, age between 50 to $70(\mathrm{OR} 3.8, P=.001)$ and age $>70$ (OR $3.8, P=.01$ ) are risk factors for in-hospital death [Sergey 2016]. Chest or back pain was the most common presenting symptom (84.8\%), often described as "sharp" (64.4\%). Back 
Table 3. Multivariable logistic regression associated with rupture

\begin{tabular}{|c|c|c|c|c|c|c|}
\hline Age & 1.029 & 1.000 & 1.059 & 0.028 & 3.835 & .050 \\
\hline Back pain & 3.322 & 1.489 & 7.901 & 1.201 & 8.101 & .004 \\
\hline Preoperative hypotension & 4.897 & 1.923 & 11.716 & 1.589 & 12.123 & .000 \\
\hline$P$ & 3.543 & 1.813 & 7.024 & 1.265 & 13.727 & .000 \\
\hline
\end{tabular}

A/G, albumin and globulin ratio; P, serum phosphorus

pain often reveals that aortic dissection involve more widely (descending aorta involved), and the aortic vessel continues to withstand the impact of blood flow, which may be a factor leading to an increased risk of rupture. In our study, back pain in the non-rupture group and rupture group are 341 (42.5\%) and $21(63.6 \%)$ with a $P$-value of .026 , while in Wu's study, they are $341(33.5 \%)$ and $50(43.1 \%)$ with a $P$-value of .051. In Wu's study, Random Forest classification of feature selection was developed, and back pain was confirmed as 'not important' [Wu 2019]. But in our study, back pain is more frequent in the rupture group and also a strong predictor with OR of $3.330(P=.003)$. This may be because of the different acute phase of patients in the two studies as pain gradually may subside over time. The occurrence of TAAD exhibits seasonally [Takagi 2018]. In our study, more haTAAD occurs in winter than other seasons and more rupture $(15 / 253,5.92 \%)$ happened in winter than other seasons $(16 / 570,2.81 \%)$.

Higher serum phosphorus level is a new predictor in aortic dissection. There are two main categories of causes of high serum phosphorus level: 1) impaired renal phosphate excretion, and 2) massive extracellular fluid phosphate loads. Most haTAAD patients reveal normal or slightly impaired renal function at admission. The leading cause of high serum phosphorus may be extensive cellular injury or necrosis in aortic tissue during aortic dissection [Chang 2017]. Several studies demonstrated that high serum phosphorus is associated with vascular calcification but also associated with calcium metabolism dysfunction or impaired renal function [Tohno 2006; Houston 2013]. But neither calcium level or renal function changes in the rupture or non-rupture groups. This may require a larger sample or further study. Low A/G often reveals liver disease or acute infection. In Wu's study, acute liver dysfunction also was confirmed as an important feature in in-hospital rupture of TAAD [Wu 2019]. In our study, both alanine transaminase and aspartate aminotransferase show no significant difference, as well as albumin and globulin. But $\mathrm{A} / \mathrm{G}$ exhibits significant difference in the rupture and non-rupture groups. $\mathrm{A} / \mathrm{G}$ maybe more sensitive in predicting rupture than other liver function tests and further study is needed in the future.

In our study, nonspecific inflammatory markers, such as WBCs and C reactive protein (CRP), do not show enough value as predictors. However, several studies do suggest
WBC and CRP as diagnostic biomarkers [Fang 2009; Ranasinghe 2010]. Our results do not support that nonspecific inflammatory markers can predict in-hospital rupture. Wu et al revealed the value of WBC count as a predictor for in-hospital rupture and also found that $\mathrm{WBC}>15 \times 10^{9} / \mathrm{L}$ is associated with a bad prognosis for TAAD patients [Wu 2019]. In Wu's study, 30 (25.9\%) in 101 rupture patients were found $\mathrm{WBC}>15 \times 10^{\%} / \mathrm{L}$ and $148(14.6 \%)$ in 1017 nonrupture patients were found $\mathrm{WBC}>15 \times 10^{\circ} / \mathrm{L}$. But in this study, six $(18.2 \%)$ in 33 rupture patients and $172(21.4 \%)$ in 803 non-rupture patients were found $\mathrm{WBC}>15 \times 10^{9} / \mathrm{L}$. That may be because in our study, patients are more severe as all patients in our study are hyperacute $(<24 \mathrm{~h})$; in Wu's study, patients are divided as with or without acute phase while "acute" was defined as 2-14 days. In addition, our patients are older in age $(53.15 \pm 13.23)$ on average than that in Wu's study $(49.5 \pm 11.7)$. These may cause the different importance of WBC level in our study. And in our center, the number and percentage of rupture is smaller, and that also may make some difference.

Bootstrapping is a method that uses random sampling with replacement. This method allows estimation of the sampling distribution of almost any statistic using random sampling methods. Bootstrap is an appropriate way to control and check the stability of the results. Bootstrapping also is a very convenient method that avoids the cost of repeating the experiment to get other groups of sample data [Hesterberg 2015]. Craiem and colleagues demonstrated that TBAD probability increases with a larger aortic arch diameter and a longer thoracic aorta, whereas threshold values increase with age. In Craiem's study, a bootstrap method was used for the internal validation of the selection of model variables and performance [Craiem 2016]. Donald and colleagues established a new aortic injury score using clinically relevant factors that incorporate patient physiology, size of the aortic lesion, and extent of the mediastinal hematoma to predict early rupture. They used bootstrap in this model for internally validation [Harris 2015]. In our study, the sample is small, and the rupture sample is much smaller. A 70:30 training and testing cohort is not appropriate in our study. In such a small data frame, bootstrap can check the stability of the final model. 
This is the first predictive nomogram for in-hospital rupture risk in haTAAD patients. It is established with six achievable and cost-effective variables that make it easy to make clinical decisions in estimating in-hospital rupture risk for haTAAD patients. The robustness of the final model was examined by repeatedly refitting the model to 1000 differently sampled training and test sets (ratio 80:20) via the bootstrap procedure. The mean AUC is 0.815 with a $95 \%$ bootstrap CI of $0.810-0.820$. These outcomes suggest that the predictive nomogram possessed necessary discriminative accuracy to predict in-hospital rupture in haTAAD patients.

\section{LIMITATIONS}

Our study has several limitations. First, it was a retrospective analysis at a single center. Second, the sample size of the rupture group is small. Finally, CTA was not available for every haTAAD patient, which could lose some imaging features associated with in-hospital rupture.

\section{CONCLUSIONS}

Advanced age, winter season, backpain, preoperative hypotension, low $\mathrm{A} / \mathrm{G}$ ratio and high serum phosphorus level are risk factors for in-hospital rupture of haTAAD.

\section{ACKNOWLEDGEMENT}

This work has been supported by the National Natural Science Foundation of China (Nos. 81970401, 81670437) and Jiangsu Provincial Key Medical Discipline (ZDXKA2016019).

\section{REFERENCES}

A WF, B JLT, C KMH, et al. 2018. Delay from Diagnosis to Surgery in Transferred Type A Aortic Dissection. American Journal of Medicine. 131(3):300-306.

Chang AR, Anderson C. 2017. Dietary Phosphorus Intake and the Kidney. Annual Review of Nutrition. 37(1):annurev-nutr-071816-064607.

Coady MA, Rizzo JA, Goldstein LJ, Elefteriades JA. 1999. Natural history, pathogenesis, and etiology of thoracic aortic aneurysms and dissections. Cardiology Clinics. 17(4):615.

Craiem D, El Batti S, Casciaro ME, et al. 2016. Age-related changes of thoracic aorta geometry used to predict the risk for acute type B dissection. International Journal of Cardiology. 654-660.
Fang L, Zhou XL, Li JJ, Hui RT. 2009. Inflammatory response is associated with aortic dissection. Ageing Research Reviews. 8(1):31-35.

Goldstone AB, Chiu P, Baiocchi M, Lingala B, Woo YJ. 2019. Interfacility Transfer of Medicare Beneficiaries With Acute Type A Aortic Dissection and Regionalization of Care in the United States. Circulation. 140(15):1239-1250

Harris DG, Rabin J, Kufera JA, et al. 2015. A new aortic injury score predicts early rupture more accurately than clinical assessment. Journal of Vascular Surgery. 61(2):332-338.

Hesterberg TC. 2015. What Teachers Should Know About the Bootstrap: Resampling in the Undergraduate Statistics Curriculum. American Statistician. 69(4):371-386.

Houston J, Smith K, Isakova T, Sowden N, Wolf M, Gutiérrez OM. 2013. Associations of Dietary Phosphorus Intake, Urinary Phosphate Excretion, and Fibroblast Growth Factor 23 With Vascular Stiffness in Chronic Kidney Disease. Journal of Renal Nutrition. 23(1):12-20.

Kageyama S, Mitake H, Nakajima A, et al. 2020. A novel risk score on admission for predicting death or need for surgery in patients with acute type A intramural hematoma receiving medical therapy. Heart and vessels. 35(8):1164-1170.

Mussa, Firas F, Horton, et al. 2016. Acute Aortic Dissection and Intramural Hematoma: A Systematic Review. JAMA: Journal of the American Medical Association.

Rampoldi V, Trimarchi S, Eagle KA, Nienaber CA, Isselbacher EM. 2007. Simple Risk Models to Predict Surgical Mortality in Acute Type A Aortic Dissection: The International Registry of Acute Aortic Dissection Score. The Annals of thoracic surgery. 83(1):55-61.

Ranasinghe AM, Bonser RS. 2010. Biomarkers in Acute Aortic Dissection and Other Aortic Syndromes. Journal of the American College of Cardiology. 56(19):1535-1541.

Sergey, Leontyev, Jean-Francois, et al. 2016. Creation of a Scorecard to Predict In-Hospital Death in Patients Undergoing Operations for Acute Type A Aortic Dissection. The Annals of thoracic surgery.

Takagi H, Ando T, Umemoto T. 2018. Meta-Analysis of Seasonal Incidence of Aortic Dissection. American Journal of Cardiology. 67(1):700-707.

Tohno Y, Tohno S, Mahakkanukrauh P, Azuma C, Maruyama H. 2006. Earlier accumulation of calcium, phosphorus, and magnesium in the coronary artery in comparison with the ascending aorta, aortic valve, and mitral valve. Biological Trace Element Research. 112(1):31-42.

Vincent B, Pauline S, Antoine GP, Stéphane L, Hervé R, Bertrand M. Impact of meteorological conditions on the occurrence of acute type A aortic dissections. Interactive Cardiovascular \& Thoracic Surgery. (3):403-406.

Wu J, Qiu J, Xie E, Jiang W, Yu C. 2019. Predicting in-hospital rupture of type A aortic dissection using Random Forest. Journal of Thoracic Disease. 11(11):4634-4646. 